\title{
Atividades farmacológicas dos flavonoides: um estudo de revisão
}

\section{Daniel Sousa dos Santos ${ }^{1}$ e Mayara Mikelle Farias Rodrigues ${ }^{2}$}

1 Mestrando do Programa de Pós-Graduação em Ciências Farmacêuticas da Universidade Federal do Amapá e graduado em Física pela Universidade Federal do Amapá. Professor do curso de Licenciatura em Educação do Campo: Agronomia e Biologia da Universidade Federal do Amapá, Brasil. E-mail: dsansousa@gmail.com

2 Mestranda do Programa de Pós-Graduação em Ciências Farmacêuticas da Universidade Federal do Amapá, Especialista em Gestão e Docência do Ensino Superior pela Faculdade de Teologia e Ciências Humanas e graduada em Farmácia pela Universidade Federal do Amapá, Brasil. E-mail: mayara.mikelle@gmail.com

RESUMO: O uso de produtos naturais como matéria-prima para a síntese de fármacos tem sido amplamente relatado ao longo do tempo. Os flavonoides, sem dúvida, são um importante grupo dentre os produtos naturais, que vem ganhando interesse especial devido apresentarem inúmeras atividades farmacológicas. O objetivo deste estudo consistiu em abordar, por meio de pesquisa bibliográfica, algumas das principais atividades farmacológicas dos flavonoides como: atividade antiviral, antitumoral, anti-inflamatória, antioxidante, e atividade hormonal e analisar os mecanismos de ação dessas substâncias.

Palavras-chave: Flavonoides; Metabólitos secundários; Atividades farmacológicas.

Pharmacological activities of flavonoids: a review study

ABSTRACT: The use of natural products as a raw material for drug synthesis has been widely reported over time. Flavonoids, undoubtedly, are an important group among natural products, which is gaining special interest due to numerous pharmacological activities. The objective of this study was to approach, through bibliographic research, some of the main pharmacological activities of flavonoids such as antiviral, antitumor, anti-inflammatory, antioxidant, and hormonal activities and to analyze the mechanisms of action these substances.

Keywords: Flavonoids; Secondary metabolites; Pharmacological activities.

\section{INTRODUÇÃO}

Os flavonoides representam um dos grupos de compostos fenólicos mais importantes e diversificados entre os produtos de origem natural. Essa classe de compostos estão amplamente distribuídos por todo o reino vegetal e até o presente são conhecidos mais de 4200 variedades de flavonoides (SIMÕES et al., 2007).

Flavonoides estão presentes em relativa abundância entre os metabólitos secundários de plantas e podem ser subdivididos em seis classes: flavonas, flavanonas, isoflavonas, flavonóis, flavanóis e antocianinas (AHERNE; O'BRIEN, 2002).
$\mathrm{Na}$ natureza podem-se encontrar flavonoides em diversas formas estruturais. Quimicamente, a maioria dos flavonoides baseia-se em uma estrutura fundamental que possui um esqueleto formado por de 15 átomos de carbono constituído por dois anéis de benzeno ( $A$ e $B$ como mostrado na Figura 1), ligado por meio de uma cadeia três carbonos entre elas e um oxigênio como heteroátomo (C) (SIMÕES et al., 2007). 
Figura 1 - Estrutura fundamental dos flavonoides Figure 1 - Fundamental structure of flavonoids

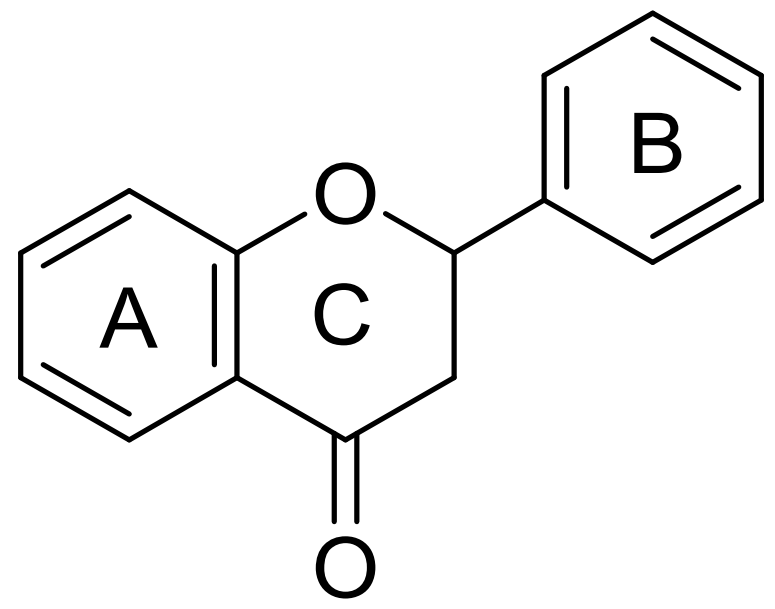

Fonte: Autor

Os flavonoides são responsáveis por inúmeras funções nas plantas. Dentre elas, podem-se mencionar a proteção contra raios ultravioleta, contra insetos, fungos, vírus e bactérias, e a capacidade de proporcionarem a atração de animais polinizadores. Além dessas características, muitos desses compostos possuem também importantes propriedades farmacológicas. Algumas são apresentadas no Quadro 1, como por exemplo: propriedades antitumoral, antiinflamatória, antiviral, antioxidante, dentre outras (AGRAWAL, 2011; GOMES et al., 2002; HEIM; TAGLIAFERRO; BOBILYA, 2002; MELLOU et al., 2005; SHASHANK; ABHAY, 2013; SELVARAJ; CHOWDHURY; BHATTACHARJEE, 2013; SIMÕES et al., 2007; FLAMBÓ, 2013).
Quadro 1 - Atividades farmacológicas de alguns flavonoides Frame 1 - Pharmacological activities of some flavonoids

\begin{tabular}{|c|c|c|}
\hline Flavonoide & $\begin{array}{l}\text { Atividade far- } \\
\text { macológica }\end{array}$ & Referência \\
\hline Acacetina & Antiviral & $\begin{array}{l}\text { CRITCHFIELD; BUTERA; } \\
\text { FOLKS, } 1996\end{array}$ \\
\hline $\begin{array}{l}\text { Amentofla- } \\
\text { vona }\end{array}$ & Antiviral & $\begin{array}{l}\text { SHUANG-CHENG et al., } \\
2001\end{array}$ \\
\hline Buteina & Anti-inflamatória & CHAN et al., 1998 \\
\hline Hesperidina & Antioxidante & OKAMURA et al., 1994 \\
\hline Quercetina & $\begin{array}{l}\text { Antitumoral } \\
\text { Anti-inflamatória } \\
\text { Antimicrobiana } \\
\text { Antioxidante }\end{array}$ & $\begin{array}{l}\text { FORMICA; REGELSON, } \\
1995 \\
\text { FERRANDIZ; ALCARAZ, } \\
1991 \\
\text { BEIL; BIRKHOLZ; } \\
\text { SEWING, } 1995\end{array}$ \\
\hline Rutina & $\begin{array}{l}\text { Anti-inflamatória } \\
\text { Antimicrobiana } \\
\text { Antioxidante } \\
\text { Antitumoral }\end{array}$ & $\begin{array}{l}\text { YOO et al., } 2014 \\
\text { PIMENTEL et al., } 2013 \\
\text { VISKUPICOVA et al., } \\
2010 \\
\text { ALONSO-CASTRO; } \\
\text { DOMÍNGUEZ; GARCÍA- } \\
\text { CARRANCÁ et al., } 2013\end{array}$ \\
\hline
\end{tabular}

Fonte: Adaptada de Simões (2007)

Devido ao grande número de flavonoides existentes na natureza, e a necessidade de avaliação de suas propriedades farmacológicas, esta revisão tem como objetivo trazer um breve estudo sobre alguns dos principais efeitos farmacológicos dos flavonoides, a fim de contribuir com estudos futuros sobre essa classe de compostos e suas aplicações.

\section{ATIVIDADES FARMACOLÓGICAS DOS FLAVONOIDES}

\subsection{Atividade antiviral}

O mecanismo de ação de flavonoides sobre a capacidade da interação do vírus desde a ligação até a sua entrada na célula hospedeira, ocorre por meio da ligação com glicoproteínas do envelope viral e receptores celulares modificando sua estrutura química e bloqueando o sitio de ligação do vírus. Em um estudo sobre a atividade contra o vírus da cinomose canina, realizado 
por Brum (2006), foi encontrada redução no título viral de $90 \%$ pelo ácido transcinâmico, $46,2 \%$ pelo ácido ferúlico, $32,48 \%$ pelo kenferol e $78 \%$ pela quercetina. Nos ensaios de atividade virucida, foi observada a redução do efeito em $99,99 \%$ (100-150 $\mu \mathrm{g} / \mathrm{mL}), 98 \%$ $(100 \mu \mathrm{g} / \mathrm{mL}), \quad 79 \% \quad(50 \mu \mathrm{g} / \mathrm{mL}), \quad$ e $96 \%$ $(100 \mu \mathrm{g} / \mathrm{mL})$, pelo ácido ferúlico, ácido transcinâmico, kaempherol e quercetina, respectivamente (BRUM, 2006).

Carvalho et al (2013) demonstraram em ensaios in vitro a atividade virucida da quercetina sobre o parvovírus canino. Para avaliação do efeito antiviral, a redução do título viral foi de $93 \%$ para concentração de $3 \mathrm{micrograma} / \mathrm{ml}$ de quercetina incubada com vírus. No mesmo estudo, foi observada também a redução do título viral na dosedependente na concentração de 1,5 micrograma/ml em testes de determinação de atividade sobre a células (CARVALHO et al, 2013).

Para investigar o mecanismo pelo qual a quercetina interage na inibição do ciclo da replicação do parvovírus canino, Carvalho et al (2013) demonstraram em teste inibitórios da adição de quercetina sobre diferentes etapas do ciclo replicativo do parvovírus, a redução de $90 \%$ do título viral na etapa de penetração, porém não teve redução significativa do título viral nas etapas de adsorção e pós-infecção. Tais resultados revelam a potencial atuação da quercetina ao interferir na ligação do vírus à célula hospedeira, dificultando a infecção pelo parvovírus canino aumentando a capacidade de seus efeitos antivirais (CARVALHO et al, 2013).

\subsection{Atividade antitumoral}

Atualmente, o interesse e o incentivo de estudos sobre novos fitocompostos com potencial efeito em células cancerígenas vem se tornando objetivo de novas propostas de pesquisa na área da saúde (ATTOUB et al., 2011).

Os flavonoides tem sido caracterizados como inibidores de células cancerosas, por apresentarem propriedades farmacológicas conhecidas como antioxidantes, possivelmente controlando assim a proliferação celular e desempenhando o bloqueio da oncogênese por mecanismos que modulam enzimas da via metabólica carcinogênica (AMADO et al., 2011; HUNG et al., 2009). Nesse sentido a atividade antitumoral de flavonoides e polifenóis passou a ser alvo de estudos com intuito de esclarecer sua interação nos mecanismo de inibição do crescimento celular por meio da apoptose em linhagens de células carcinogênicas (JADVAR; ALAVI; GAMBHIR, 2009).

Brito et al. (2015) verificaram a capacidade que a quercetina possui ao inibir a proliferação de linhagem celular de carcinoma hepatocelular, revelando que a quercetina consegue inibir a função do transportador de glicose (GLUT-1).

Em um estudo realizado por Pereira et al (2015), foi investigado a indução de morte celular por apoptose em linhagens de célula de câncer de pulmão (H4600), no qual revelou a redução do crescimento tumoral e indução de morte celular mediante o tratamento com flavonoide morina, na concentração de 800 micrograma $/ \mathrm{ml}$. Avaliados pelos ensaios testes de MTT, por microscopia de fluorescência e citometria de fluxo (PEREIRA et al., 2015).

\subsection{Atividade anti-inflamatória}

A atividade anti-inflamatória dessa classe de substâncias vem sendo investigada devi-

https://periodicos.unifap.br/index.php/estacao Macapá, v. 7, n. 3, p. 29-35, set./dez. 2017 
do ao poder que alguns compostos fenólicos possuem em inibir as vias de ciclooxigenase (COX) e lipoxigenase, as quais desempenham um papel importante como mediadores inflamatórios (SIMÕES et al., 1988; FERRANDIZ; ALCARAZ, 1991).

Segundo Moroney et al. (1988), as enzimas COX e 5-lipoxigenase estão envolvidos na libertação de ácido araquidônico, o qual está envolvido na formação de leucotrienos que é um ponto de partida para uma resposta inflamatória geral. Os compostos fenólicos santina e 6-hidróxi-canferol-3,6dimetil-éter, derivados do kenferol e isolados de Tanacetum parthenium Sch. Bip. foram capazes de inibir a COX e 5- lipoxigenase (MORONEY et al., 1988).

Efeitos anti-inflamatórios in vivo e in vitro aparecem na literatura para os compostos diosmina e hesperidina, de forma que a atividade anti-inflamatória tem sido atribuída à inibição da síntese de prostagladinas PGE2 e PGE2a e à atividade antioxidante (GARG et al., 2001).

Outro composto que apresentou uma eficaz ação anti-inflamatória foram as antocianinas por meio da inibição de liberação e de síntese de substâncias endógenas, que promovem inflamação como a histamina. Por este efeito as antocianinas apresentam forte ação antialérgica (AMELLAL et al., 1985).

\subsection{Atividade antioxidante}

Flavonoides apresentam atividade antioxidante pelo fato de serem quelantes de metais, varredores de radicais livres, inclusive o radical hidroxila ou radical peróxido, além de neutralizarem espécies oxidantes como o ânion superóxido $\left(\mathrm{O}^{2-}\right)$ e atuarem em sinergismo com outros antioxidantes como as vitaminas C e E. Atualmente, estuda-se a respeito da estrutura dos flavonoides e a ação antioxidante. Essa atividade antioxidante também está ligada a inibição de enzimas ciclo, lipoxigenase, NADPHoxidade, xantina-oxidase e fosfolipase, e estimulação de enzimas com atividade antioxidante como a catalase e a superóxidodismutase (TRUEBA; SÁNCHEZ, 2001).

A quercetina pertencente ao grupo dos flavonóis é um composto que tem chamado atenção devido seus efeitos antioxidantes. Estudos realizados por Knekt et al., (2000) mostraram que a quercetina, ao ser ingerida, não é inteiramente absorvida no tubo digestivo, e os metabólitos formados contribuem para alterar o efeito antioxidante da quercetina. Ainda nesse estudo, foi analisado o efeito da quercetina e a ocorrência da doença cerebrovascular, com grande número grande de pacientes, 9.208 indivíduos, entre os anos de 1966 e 1972. Os resultados sugeriram que a ingesta de maçã está relacionada à diminuição no risco de doença cérebrovascular de origem trombótica. Todavia, não foi encontrada uma associação quando a quercetina foi analisada isoladamente (KNEKT et al., 2000).

\subsection{Atividade hormonal}

Os flavonóides também demonstram ter atividades reguladoras sobre hormônios por ligação a esteróides 17-beta-hidroxi desidrogenases, que regulam os níveis de estrogênio e de androgênio nos humanos, e ao esteróide 3-beta-hidroxi desidrogenases, os quais demonstram propriedades reguladoras dos níveis de progestina e androgêno em seres humanos. Estudos relatam existir evidências de que as isoflavonas previnem a fragilidade óssea. A ipriflavona, classe de 
isoflavona sintética, contribui na manutenção da densidade óssea em mulheres que se encontram na fase pré-menopausa e mantém ou até mesmo aumentam a densidade óssea em mulheres na fase pósmenopausa (KNIGHT; EDEN, 1995).

No mercado farmacêutico brasileiro, existem fármacos contendo a ipriflavona para a reposição hormonal ou para o tratamento e prevenção da osteoporose, diminuindo a liberação de cálcio ósseo e a reabsorção óssea (SIMÕES, 2007).

\section{CONCLUSÃO}

O presente estudo abordou por meio de revisão de literatura algumas atividades farmacológicas dos flavonoides e seus derivados, relatando os possíveis mecanismos de ação dessas substâncias que subsidiam tais atividades. Mesmo que, ainda sejam necessários estudos clínicos para melhor esclarecer os mecanismos de ação e os efeitos benéficos deste grupo de substâncias, os dados da literatura levantados neste estudo indicam suas funções e sugerem aplicações promissoras, as quais apontam para o potencial emprego clínico desses metabólitos no futuro.

\section{REFERÊNCIAS}

AGRAWAL, A. D. Pharmacological activities of flavonoids: a review. International Journal of Pharmaceutical Sciencies and Nanotechnology, v. 4, p. 1394-1398, 2011.

AHERNE, S. A.; O'BRIEN, N. M. Dietary flavonols: Chemistry, food content, and metabolism. Nutrition, v. 18 , n. 1 , p. $75-81$, 2002.

ALONSO-CASTRO, A. J.; DOMÍNGUEZ, F.; GARCÍA-CARRANCÁ, A. Rutin exerts anti- tumor effects on nude mice bearing SW480 tumor. Archives of Medical Research, v. 44, p. 346-351, 2013.

AMADO, N. G. et al. Flavonoids: potential wnt/beta-catenin signaling modulators in cancer. Life Sci. v. 89, n. 15-16, p. 545-554, 2011.

AMELLAL, M. et al. Inhibition of mast cell histamine release by flavonoids and biflavonoids. Planta Med., v. 51, n. 1, p. 1620, 1985.

ATTOUB, S. et al. Inhibition of cell survival, invasion, tumor growth and histone deacetylase activity by the dietary flavonoid luteolin in human epithelioid cancer cells. Eur. J. Pharmacol. v. 651, p. 18-25, 2011. BEIL, W.; BIRKHOLZ, C.; SEWING, K. F. Effects of flavonoids on parietal cell acid secretion, gastric mucosal prostaglandin production and Helicobacter pylori growth. Arzneimittelforschung. v. 45, n. 6, p. 697700, 1995.

BRITO, A. F. et al. Acção anti-cancerígena da quercetina no carcinoma hepatocelular: o papel do GLUT-1," Rev. Port. Cir., n. 25, p. 798-806, 2015.

BRUM, L. P. Atividade antiviral dos compostos fenólicos (ácidos ferúlico e transcinâmico) e dos flavonoides (quercetina e kaempherol) sobre os herpesvirus bovino 1 , herpesvírus bovino 5 e vírus da cinomose canina. 2006. Tese de doutorado (Doutorado em Bioquímica Agrícola), Universidade Federal de Viçosa, Viçosa, Minas Gerais, 2006.

CARVALHO, O. V. et al. Potencial antiviral da quercetina sobre o parvovírus canino. Arq. Bras. Med. Vet. Zootec. v.65, n.2, p. 353358, Belo Horizonte, 2013.

CHAN, S. C. et al. Three new flavonoids and antiallergic, anti-inflammatory constituents from the heartwood of Dalbergia odorifera. 
Planta Med., v. 64, n. 2, p. 153-158, 1998. CRITCHFIELD, J. W.; BUTERA, S. T.; FOLKS, T. M. Inhibition of HIV activation in latently infected cells by flavonoid compounds. AIDS Res. Hum. Retroviruses, v. 12, n. 1, p. 39-46, 1996.

FERRANDIZ, M. L: ALCARAZ, M. J. Antiinflammatory activity and inhibition of arachidonic acid metabolism by flavonoids. Agents Actions, v. 32, n. 3-4, p. 283-288, 1991.

FLAMBÓ, D. F. A. L. P. Atividades biológicas dos flavonoides: atividade antimicrobiana. 2013. Dissertação (Mestrado em Ciências Farmacêuticas), Faculdade de Ciências da Saúde, Universidade Fernando Pessoa, Porto, 2013.

FORMICA, J. V.; REGELSON, W. Review of the biology of quercetin and related bioflavonoids. Food and Chemical Toxicology. v. 33, n. 12. p. 1061-1080, 1995.

GARG, A. et al. Chemistry and pharmacology of the Citrus bioflavonoid hesperidin. Phytotherapy Research. v. 15, n. 8. p. 655669, 2001.

GOMES, S. M. et al. Efeito de diferentes doses de flavonóides, Rev. Nutr., v. 15, n. 1, pp. 45-51, 2002.

HEIM, K. E.; TAGLIAFERRO, A. R.; BOBILYA, D. J. Flavonoid antioxidants: Chemistry, metabolism and structure-activity relationships. Journal of Nutritional Biochemistry, v. 13, n. 10, p. 572-584, 2002.

HUNG, J. Y. et al. Didymin, a dietary flavonoid glycoside from citrus fruits, induces fas-mediated apoptotic pathway in human non-small-cell lung cancer cells in vitro and in vivo. Lung Cancer. v. 68, n. 3, p. 366-374, 2009.

JADVAR, H.; ALAVI, A.; GAMBHIR, S. S. 18FFDG uptake in lung, breast, and colon cancers: molecular biology correlates and dis- ease characterization. J. Nucl. Med., v. 50, n. 11, p. 1820-7, 2009.

KNEKT, P. et al. Quercetin intake and the incidence of cerebrovascular disease. Eur. J. Clin. Nutr., v. 54, n. 5, pp. 415-417, 2000.

KNIGHT, D. C.; EDEN, J. A. Phytoestrogens a short review. Maturitas, v. 22, n. 3. p. 167-175, 1995.

MELLOU, F. et al. Biocatalytic preparation of acylated derivatives of flavonoid glycosides enhances their antioxidant and antimicrobial activity. J. Biotechnol., v. 116, n. 3, p. 295-304, 2005.

MORONEY, M. A. et al. Selectivity of neutrophil 5-lipoxygenase and cyclo-oxygenase inhibition by an anti-inflammatory flavonoid glycoside and related aglycone flavonoids. J. Pharm. Pharmacol., v. 40, n. 11, p. 787-792, 1988.

OKAMURA, N. et al. Flavonoids in Rosmarinus officinalis leaves. Phytochemistry, v. 37, n. 5, p. 1463-1466, 1994.

PEREIRA, W. L. et al. Ação antiproliferativado flavonoide morina e do extrato da folha de oliveira (Oleaeuropaea L.) contra a linhagem de célula H460. Rev. Bras. PI. Med., v. 17, n. 4, p. 798-806, 2015.

PIMENTEL, R. B. D. Q. et al. Antimicrobial activity and rutin identification of honey produced by the stingless bee Melipona compressipes manaosensis and commercial honey. BMC Complementary and Alternative Medicine, v. 13, p. 151, 2013.

SELVARAJ, K.; CHOWDHURY, R.; BHATTACHARJEE, C. Isolation and structural elucidation of flavonoids from aquatic fern Azolla microphylla and evaluation of free radical scavenging activity. Int. J. Pharm. Pharm. Sci., v. 5, n. 3, p. 743-749, 2013.

SHASHANK, K.; ABHAY, K. Review article chemistry and biological activities of flavonoids: an overview. Sci. World J, v. 4, n. 2, 
p. 32-48, 2013.

SHUANG-CHENG, M. A. Antiviral amentoflavone from Selaginella sinensis. Biological \& Pharmaceutical Bulletin, v. 24, n. 3. p. 311-312, 2001.

SIMÕES, C. M. O. et al. (Org.). Famacognosia: da planta ao medicamento. 6. ed. Porto Alegre: Editora da UFSC e UFRGS, 2007. SIMÕES, C. M. O. et al. Pharmacological investigations on Achyrocline satureioides (Lam.) DC., compositae. J. Ethnopharmacol, v. 22, n. 3, p. 281-293, 1988.

TRUEBA, G. P.; SÁNCHEZ, G. M. Los flavonoides como antioxidantes naturales. Acta Farm. Bonaerense. v. 20, n. 4, p. 297-306, 2001.

VISKUPICOVA, J. et al. Lipophilic rutin derivatives for antioxidant protection of oilbased foods. Food Chemistry, v. 123, p. 4550, 2010.

YOO, H. et al. Anti-inflammatory effects of rutin on HMGB1-induced inflammatory responses in vitro and in vivo. Inflammation Research, v. 63, p. 197-206, 2014.

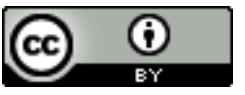
License information: This is an openaccess article distributed under the terms of the Creative Commons Attribution License, which permits unrestricted use, distribution, and reproduction in any medium, provided the original work is properly cited.

Artigo recebido em 26 de outubro de 2017.

Avaliado em 06 de novembro de 2017.

Aceito em 06 de novembro de 2017.

Publicado em 19 de dezembro de 2017.

\section{Como citar este artigo (ABNT):}

SANTOS, Daniel Sousa dos; RODRIGUES, Mayara Mikelle Farias. Atividades farmacológicas dos flavonoides: um estudo de revisão. Estação Científica (UNIFAP), Macapá, v. 7, n. 3, p. 29-35, set./dez. 2017. 